\section{FRI0067 TREATMENT WITH ANAKINRA REDUCES THE RATE OF JOINT DESTRUCTION AND SHOWS ACCELERATED BENEFIT IN THE SECOND 6 MONTHS OF TREATMENT FOR PATIENTS WITH RHEUMATOID ARTHRITIS}

${ }^{1} \mathrm{HK}$ Genant, ${ }^{2} \mathrm{~B}$ Bresnihan, ${ }^{3} \mathrm{E} N \mathrm{Ng},{ }^{3} \mathrm{~S}$ Robbins, ${ }^{3} \mathrm{RD}$ Newmark, ${ }^{3} \mathrm{D}$ McCabe. ${ }^{1}$ Department of Radiology, University of California, San Francisco, USA; ${ }^{2}$ Department of Rheumatology, St. Vincent's University Hospital, Dublin, Ireland; ${ }^{3}$ Clinical Development, Amgen Inc., Thousand Oaks, USA

\subsection{6/annrheumdis-2001.1196}

\section{Background}

Objectives Evaluate the effect of anakinra treatment on joint destruction in 1) patients completing a previous 6 months of anakinra treatment, and 2) patients completing 24 weeks of placebo.

Methods 472 Patients with RA entered a 24 week, randomised clinical trial and received placebo or anakinra 30,75 or $150 \mathrm{mg}$ daily. On completing this phase, patients receiving placebo were re-randomised to one of the treatment groups and patients receiving anakinra continued treatment for a further 24 week extension study. Radiographs, obtained at 0, 24 and 48 weeks, were evaluated for joint damage using a modified Sharp method (Genant AJR '00).

Results For those patients who rolled over to treatment with anakinra after 6 months of placebo, all three anakinra dose groups showed significant reduction in the rate of joint destruction. Patients who received the same dose of anakinra for the entire 12 months showed an accelerated benefit by slowing radiographic progression in the second 6 months $(75$ and 150 $\mathrm{mg})$.

\begin{tabular}{|c|c|c|c|c|}
\hline & $30 \mathrm{mg}$ & $75 \mathrm{mg}$ & $150 \mathrm{mg}$ & All \\
\hline $\begin{array}{l}\text { Change on placebo } \\
0-6 \text { mo }\end{array}$ & 2.55 & 2.01 & 5.79 & 3.43 \\
\hline $\begin{array}{l}\text { Change on anakinra } \\
6-12 \mathrm{mo}\end{array}$ & 1.10 & 0.88 & 1.99 & 1.31 \\
\hline $\begin{array}{l}\text { Difference placebo - } \\
>\text { anakinra }\end{array}$ & $\begin{array}{l}-1.46(n=19) \\
p=0.008\end{array}$ & $\begin{array}{l}-1.13(n=20) \\
p=0.010\end{array}$ & $\begin{array}{l}-3.81(n=19) \\
p=0.002\end{array}$ & $\begin{array}{l}-2.11(n=58) \\
p<0.001\end{array}$ \\
\hline $\begin{array}{l}\text { Change on anakinra } \\
0-6 \text { mo }\end{array}$ & 1.74 & 1.95 & 1.78 & 1.82 \\
\hline $\begin{array}{l}\text { Change on anakinra } \\
6-12 \mathrm{mo}\end{array}$ & 1.61 & 0.95 & 0.85 & 1.18 \\
\hline $\begin{array}{l}\text { Difference anakinra } \\
\text { - > anakinra }\end{array}$ & $\begin{array}{l}-0.13(n=68) \\
p=0.282\end{array}$ & $\begin{array}{l}-1.00(n=61) \\
p=0.006\end{array}$ & $\begin{array}{l}-0.93(n=49) \\
p=0.008\end{array}$ & $\begin{array}{l}-0.65(n= \\
\text { 178) } p<0.001\end{array}$ \\
\hline
\end{tabular}

Conclusion In a 24 week study of RA, anakinra significantly reduced the rate of progressive joint damage. When treatment was maintained for a further 24 weeks, the rate of progressive joint damage was significantly slower compared to the first 24 week period.
FRI0068 102-WK CLINICAL AND RADIOLOGIC RESULTS FROM THE ATTRACT TRIAL: A 2 YEAR, RANDOMISED, CONTROLLED, PHASE 3 TRIAL OF INFLIXIMAB $\left(\right.$ REMICADE $^{\circledR}$ ) IN PTS WITH ACTIVE RA DESPITE MTX

${ }^{1} \mathrm{R}$ Maini, ${ }^{2} \mathrm{D}$ Van der Heijde, ${ }^{3} \mathrm{~J}$ Smolen, ${ }^{4} \mathrm{~J}$ Kalden, ${ }^{5} \mathrm{~F}$ Breedveld, ${ }^{6} \mathrm{P}$ Emery, ${ }^{7}$ E Keystone, ${ }^{8} \mathrm{G}$ Harriman, ${ }^{9} \mathrm{P}$ Lipksy. ${ }^{1}$ Rheumatology, Kennedy Institute of Rheumatology, Hammersmith; ${ }^{2}$ Rheumatic Diseases, ATTRACT Investigator, Maastricht; ${ }^{3}$ Medicine, ATTRACT Investigator, Vienna; ${ }^{4}$ Clin Immun \& Rheum, ATTRACT Investigator, Erlangen; ${ }^{5}$ Rheumatology, ATTRACT Investigator, Leiden, EU; ${ }^{6}$ Rheumatology, ATTRACT Investigator, Leeds, UK; ${ }^{7}$ Rheumatology, ATTRACT Investigator, Toronto; ${ }^{8}$ Clinical Research, Centocor, Malvern; ${ }^{9}$ Rheumatology, ATTRACT Investigator, Bethesda, USA

\subsection{6/annrheumdis-2001.1197}

Background The ATTRACT trial studied the long term efficacy and tolerability of infliximab in 428 pts with active RA despite MTX (median dose of $15 \mathrm{mg} / \mathrm{wk}$ ).

Objectives

Methods While continuing MTX, pts were randomly assigned to one of five therapeutic arms in which infliximab at 3 or $10 \mathrm{mg} /$ $\mathrm{kg}$ or placebo infusions were given at 0,2 , and 6 wks and every 4 or 8 wks thereafter. Clinical benefit was assessed by maintenance of at least an ACR20 level of response. Using the van der Heijde modified Sharp system assessing bone erosions and joint space narrowing, the total radiologic score (TRS) was calculated for each patient at $0,30,54$, and 102 wks. Adverse events were tabulated.

Results Infliximab in combination with MTX is well tolerated and results in a sustained clinical benefit at all doses and frequencies over 2 years. Additionally, radiologic progression was essentially arrested in all infliximab plus MTX treatment arms as compared to the MTX control group. All infliximab dose regimens were comparable in clinical and radiologic benefit.

\begin{tabular}{|c|c|c|c|c|c|c|c|}
\hline & Placebo & $\begin{array}{l}3 \mathrm{mg} / \\
\mathrm{kg} \\
\text { q } 8 \\
\text { wks }\end{array}$ & $\begin{array}{l}3 \text { mg/ } \\
\text { kg } \\
\text { q } 4 \\
\text { wks }\end{array}$ & $\begin{array}{l}10 \mathrm{mg} / \\
\mathrm{kg} \\
\mathrm{q} 8 \\
\text { wks }\end{array}$ & $\begin{array}{l}10 \mathrm{mg} / \\
\mathrm{kg} \\
\text { q } 4 \\
\text { wks }\end{array}$ & $\begin{array}{l}\text { Total } \\
\text { Infliximab }\end{array}$ & $\begin{array}{l}p- \\
\text { value }\end{array}$ \\
\hline ACR20 & 16 & 41 & 40 & 48 & 42 & 43 & $<0.001$ \\
\hline $\begin{array}{l}\text { Change in } \mathrm{HAQ} \\
\text { Mean }\end{array}$ & 0.3 & 0.4 & 0.5 & 0.5 & 0.4 & 0.5 & $<0.001$ \\
\hline $\begin{array}{l}\text { Change in HAQ } \\
\text { Median }\end{array}$ & 0.1 & 0.3 & 0.3 & 0.4 & 0.3 & 0.4 & $<0.001$ \\
\hline $\begin{array}{l}\text { Change in TRS } \\
\text { Mean }\end{array}$ & 12.6 & 1.0 & 1.0 & 1.1 & -0.4 & 0.7 & $<0.001$ \\
\hline $\begin{array}{l}\text { Change in TRS } \\
\text { Median }\end{array}$ & 4.3 & 0.4 & 0.5 & 1.0 & 0.0 & 0.5 & $<0.001$ \\
\hline $\begin{array}{l}\% \text { pts }>=1 \\
\text { serious } A E\end{array}$ & $33 \%$ & $33 \%$ & $23 \%$ & $29 \%$ & $32 \%$ & $29 \%$ & 0.603 \\
\hline
\end{tabular}

No unexpected adverse events, nor an increase in any previously observed side effect were reported.

Conclusion Infliximab in combination with MTX is well tolerated and results in a sustained clinical benefit at all doses and frequencies over 2 years. Additionally, radiologic progression was essentially arrested in all infliximab plus MTX treatment arms as compared to the MTX control group. All infliximab dose regimens were comparable in clinical and radiologic benefit. 